\title{
20 Tonträger und Musikmedien: Zur Rhetorik technischer Schallproduktion vom Phonographen zum MP3-Spieler
}

\begin{abstract}
This chapter analyses the rhetorical functions of sound recording media and playback devices from the phonograph to the MP3 player. Following the history of technical sound reproduction, the authors show how the materials, the design and the sonic characteristics of changing technologies influenced cultural constructions of meaning and shaped the media practices that were bound up with them. In the first half of the $20^{\text {th }}$ century manufacturers used product design and advertising to establish both the phonograph and the gramophone as pieces of bourgeois musical furniture coequal to the piano, while the design and material composition of radio sets responded to new public media spheres and standardised mass production. The socio-economic upheavals after the Second World War, marked by progressive individualisation and an increased consumerism, also resulted in the dissemination of new sound carriers, such as the vinyl record and the music cassette, as well as modular stereo systems that featured a considerably improved sound quality. This chapter closes with reflections on the digitisation of music culture and discusses controversies surrounding the purportedly "cold" sound of digital reproduction are presented in order to illustrate how rhetorics on sonic signatures continue to shape questions of social and cultural distinction to the present day.
\end{abstract}

Stichwörter: Tonträger, Musikreproduktion, Phonograph, Grammophon, Radio, Schallplatte, Stereoanlage, Audiokassette, Walkman, Compact Disc, MP3, High Fidelity

Keywords: Recorded Music, technologies of sound reproduction, phonograph, gramophone, radio, record, stereo system, audiocassette, Walkman, compact disc, MP3, high fidelity

Gliederung: 1 Einleitung - 2 Phonograph und Grammophon - 3 Radio - 4 Langspielplatte, Single und Stereoanlage - 5 Musikkassette: Mobilität und Mixtape-Kultur - 6 Von der Compact Disc zu Mp3: Die Digitalisierung der Musikkultur - 7 Fallbeispiel: Der „kalte“ Klang des Digitalen - 8 Literatur

\section{Einleitung}

Akustische Medien haben seit ihrem Aufkommen im letzten Viertel des 19. Jahrhunderts die auditive Kultur im Allgemeinen und die Produktion, Distribution und Rezeption von Musik im Besonderen entscheidend verändert. Dieser Beitrag befasst sich 
mit den rhetorischen Funktionen von Tonträgern und Musikmedien - von frühen analogen Geräten wie dem Phonographen und dem Grammophon bis hin zur digitalen Musikwiedergabe mittels CD-Spieler, Mp3-Spieler oder Smartphone. Einem chronologischen Aufbau folgend, beziehen wir uns jeweils auf drei verschiedene Aspekte von Rhetorik:

1. auf die Rhetorik von Tonträgern, denn Trägermedien wie Wachswalze, Schellackplatte, Langspielplatte, Audiokassette oder Compact Disc wurden für spezifische Verwendungsweisen konzipiert, die sich jeweils in der Form bzw. dem Design des Tonträgers wiederspiegeln. Im Laufe der Mediengeschichte sind jedoch auch viele Nutzungspraktiken entstanden, die von ihren Entwicklern gar nicht vorhergesehen wurden. Ebenso unterscheiden sich Tonträger hinsichtlich der kulturellen Bedeutungen, die mit ihnen verbunden werden.

2. auf die Rhetorik der Musikmedien, denn neben medialen Eigenschaften der Tonträger war vor allem die Funktionalität und die Gestaltung von Wiedergabegeräten im Hinblick auf Formgebung und Materialauswahl prägend für die Ausbildung von Nutzungskontexten. Ein Musikgerät als Objekt im Raum kommuniziert Botschaften durch seine Form, die Farbgebung und die verwendeten Materialien.

3. auf die Diskursrhetorik über den Klang von Tonträgern und Musikmedien. Die Möglichkeit, Klang technisch zu speichern und wiederzugeben, führte immer wieder $\mathrm{zu}$ den unterschiedlichsten Kontroversen, etwa über klangtreue Schallwiedergabe oder den Wert von „Konservenmusik“ im Vergleich zu Konzertaufführungen. ${ }^{1}$ Solche Debatten begleiteten nicht nur die Frühzeit der technischen Schallreproduktion, sondern entbrannten in den frühen 1980er Jahren erneut, als die Elektronikkonzerne Philips und Sony im Verbund mit der Musikindustrie begannen, mit der Einführung der Compact Disc die Digitalisierung der Musikwiedergabe voranzutreiben. Hiermit beschäftigt sich das Fallbeispiel des Beitrags.

\section{Phonograph und Grammophon}

Vor 1900 war das Musikhören im häuslichen Umfeld vorwiegend an das - zumeist gemeinsame - Musizieren und Singen gebunden. Insbesondere in bürgerlichen Schichten nahm „Hausmusik“ einen hohen Stellenwert im familiären wie gesellschaftlichen Leben ein. Im Laufe des 19. Jahrhunderts entwickelte sich das Klavier zum bevorzugten Musikinstrument des Bürgertums, da es sich besonders gut als Begleitinstrument eignete, gleichzeitig aber auch in den Gesellschaftsräumen eine Signalfunktion übernahm und - nicht zuletzt aufgrund der Größe und der verhältnismäßig hohen Anschaffungskosten - die ökonomischen Verhältnisse seiner Besit-

1 Vgl. etwa Adorno 1934/1984; Rzehulka 1986. 
zer repräsentierte. Das Klavier war Musikinstrument und Möbelstück in einem und wurde, etwa durch aufwendige Verzierungen, an den vorherrschenden Einrichtungsstil angepasst. ${ }^{2}$ Technische Musikmedien veränderten die häuslichen Hörpraktiken fundamental: Phonograph und Grammophon machten ein aktives Musizieren überflüssig und brachten zudem ganze Orchester, gefeierte Opernsänger und Chöre im Haus zum Erklingen.

In seinem Buch Nadel, Rille, Trichter hat Stefan Gauß die Frühgeschichte phonographischer Medien rekonstruiert und deren technische und ästhetische Entwicklung, Vermarktung und Nutzung in Deutschland aus kulturwissenschaftlicher Perspektive nachgezeichnet. ${ }^{3}$ Durch den von Thomas Alva Edison im Dezember 1877 vorgestellten Phonographen war es erstmals möglich, komplexe Schallereignisse - wie beispielsweise die menschliche Stimme - in ihrem zeitlichen und spektralen Verlauf zu speichern und zu einem späteren Zeitpunkt oder an einem anderen Ort zu reproduzieren. Die Besonderheit des Phonographen bestand dabei in seiner relativen funktionalen Offenheit, d.h. in der Möglichkeit, Schallereignisse aller Art sowohl aufzeichnen als auch wiedergeben zu können. Es ist daher kein Zufall, dass der Gebrauchszweck des Phonographen noch nicht von Anfang an auf das Abspielen von Musik ausgelegt war (diese Nutzungsweise hat sich erst nach und nach herauskristallisiert).

Für Edison stand zunächst die Aufnahmefunktion des Phonographen und damit seine wörtliche Bedeutung als Schall- oder Klangschreiber im Vordergrund. In einem Beitrag für die Zeitschrift The North American Review skizzierte Edison eine Reihe möglicher kommerzieller Verwendungsweisen des Phonographen: ${ }^{4}$ Dieser sollte als Diktiermaschine etwa für Geschäftskorrespondenzen das Briefeschreiben und -lesen ersetzen. Der „Briefeschreiber“ konnte, nachdem er seine Walze an einen Empfänger verschickt hatte, gleichzeitig eine Aufnahme für sich behalten, weil eine Walze - ähnlich einem Schreibmaschinendurchschlag - auch mit zwei Stanniolfolien versehen werden konnte. An zweiter Stelle schwebte Edison die Produktion phonographischer Bücher vor, die zum einen die ehrenamtlichen oder professionellen Vorleser ersetzen sollten, die üblicherweise in Hospitälern oder Blindenhäusern vorlasen, und zum anderen als Lehrmittel zum Erlernen von Sprachen oder der Vortragskunst zum Einsatz kommen sollten. Edison stellte sich zudem vor, Stimmenarchive von Familienmitgliedern und berühmten Persönlichkeiten anzulegen, um menschliche Stimmen über ihren Tod hinaus zu konservieren. Erst nach diesen auf die Speicherung von gesprochener Sprache ausgerichteten Anwendungen weist Edison auf die Möglichkeit hin, den Phonographen zur Wiedergabe von Liedern und Musik zu nutzen, die der Unterhaltung dienen, aber auch den Musikunterricht erleichtern sollten. Weitere

2 Vgl. Sabin 1998, 28.

3 Siehe Gauß 2009.

4 Vgl. Edison 1878. 
Anwendungsszenarien bilden tönende Spielzeuge, sprechende Puppen, automatische Zeitansagen und die Möglichkeit, Telefongespräche aufzuzeichnen. ${ }^{5}$

Abgesehen vom sprechenden Spielzeug steht die Nutzung der Walze vor allem im Zusammenhang mit Rationalisierungsmaßnahmen, die die menschliche Arbeit überflüssig machen sollte. Edisons Visionen waren weitsichtig, ließen sich aber - vor allem aufgrund der mangelhaften Tonqualität und einer damit verbundenen schlechten Verständlichkeit - kaum realisieren und wirtschaftlich vermarkten. Die meisten Anwendungen, wie etwa der Anrufbeantworter, wurden erst später und auf der Basis elektronischer Tontechnik realisiert. Ein Bereich, in dem der Phonograph als akustisches Speichermedium tatsächlich eine große Anwendung fand, war der Gebrauch innerhalb der ethnologischen und linguistischen Forschung, die mit dem Phonographen die Hoffnung verband, vom Aussterben bedrohte Sprachen und Mundarten archivieren und so der Nachwelt erhalten zu können. ${ }^{6}$

Entsprechend der Edison'schen Vorstellung vom Phonographen als „Sprechmaschine“ wurde auch die äußere Gestalt des Phonographen in erster Linie als Arbeitsgerät für das Bürowesen konzipiert. So erinnerten Edisons Modell des „Perfected Phonograph“ aus dem Jahr 1888 ebenso wie das Konkurrenzmodell „Graphophon“ von Charles Sumner Tainter und Alexander und Chichester Bell optisch an eine Dampfbzw. Nähmaschine. Doch als Büromaschine konnte sich der Phonograph nicht etablieren, da die Handhabung zu umständlich war, um damit tatsächlich Zeit und Personal einsparen zu können. Einen kommerziellen Erfolg erzielte der Phonograph erst um 1890 mit den sogenannten „Nickel-in-the-Slot-Machines“.7 Anstelle gesprochener Botschaften nutzte man die Walzentonträger zur Reproduktion von Musikaufnahmen, die an öffentlichen Orten wie Jahrmärkten, Tanzlokalen oder Hotelfoyers gegen Münzgeld angehört werden konnten. Zu diesem Zweck erhielt der Phonograph ein Holzgehäuse, das ihn vor dem Zugriff der Hörer schützte. Die Mechanik, üblicherweise durch ein Glasfenster sichtbar, war Teil der Unterhaltung und forderte die Menschen geradezu auf, den Phonographen in Gang zu setzen. Die Musik wurde über Hörschläuche zum Ohr geleitet, damit nur zahlende Kunden in den Genuss der etwa zweiminütigen Musikstücke kamen. Das Prinzip der Jukebox war entstanden: ein Apparat, der den Verkauf von Musik als immaterielle Ware ermöglichte. Da diese Nutzungsweise großen Anklang fand, begannen die Hersteller von Münzphonographen, auch Geräte für den Hausgebrauch zu bauen.

Parallel zum Phonographen entstand mit dem von Emile Berliner entwickelten Grammophon ein ernstzunehmendes Konkurrenzprodukt auf dem erblühenden Markt für technische Musikreproduktion. Im Gegensatz zum Phonographen verwen-

5 Vgl. Edison 1878, 232-235.

6 Zur problematischen Geschichte des Phonographen als Forschungsinstrument der Ethnologie vgl. Stangl 2000; Sterne 2003, 311-333.

7 Vgl. Gitelman 2006, 44-48. 
dete Berliner bei seinem Gerät keine Walzen, sondern kreisförmige Wachsplatten als Tonträger; die Klangaufzeichnung und -wiedergabe geschah im Seitenschriftverfahren. Wie die Bezeichnung Grammophon (wörtlich „Schrifttöner“) bereits nahe legt, stand für Berliner, der auf die Vermarktung von Musik zielte, von Beginn an die Wiedergabefunktion des Geräts im Vordergrund. So verfügten auch die meisten Grammophone über keine Aufnahmefunktion. Um die Jahrhundertwende entstand in den USA und Europa eine florierende Phonoindustrie, die sowohl die Apparate als auch die Tonträger produzierte und zunehmend Künstlerinnen und Künstler für Musikaufnahmen verpflichtete. Um 1901 gab es allein in Deutschland 50 Betriebe, die Grammophone herstellten; 1907 waren es bereits $181 .^{8}$ Die Zahl verkaufter Schallplatten belief sich im selben Jahr allein in Deutschland auf 18 Millionen Stück. ${ }^{9}$

Grammophone bestanden aus zwei markanten Teilen, der Schatulle, auf der die Platte abgespielt wurde und in der sich der Antrieb befand, und dem Trichter. Weil die Technik bei allen Modellen ähnlich war und sich nicht schnell änderte, entschied sich die Wahl für ein bestimmtes Modell häufig allein aufgrund der Optik der Geräte. Es entstand eine große Vielfalt an Gehäuse- und Trichterformen, für die teilweise sehr ungewöhnliche Materialien, darunter Pappmaché, Glas oder Aluminium, verwendet wurden. Neben dem ursprünglichen Trompetentrichter aus Metall gab es Trichter in Glocken- und Blumenform, die sich aus mehreren Lamellen zusammensetzten, farbig angemalt oder mit Holz imitierender Folie beklebt wurden. Besonders beliebt waren Trichter aus Eichen-, Mahagoni- oder Nussbaumholz, weil sowohl unter Konstrukteuren wie Käufern das Vorurteil verbreitet war, dass Holz - ähnlich wie bei Musikinstrumenten - aufgrund seiner Resonanzeigenschaften einen guten Klang liefern würde. ${ }^{10}$ Die starke Ausdifferenzierung an Grammophonmodellen kann aber auch anders gedeutet werden: Mit der Reproduktion von Musik ging der visuelle Aspekt der Aufführung verloren, der bis dahin mit der Musikrezeption verbunden war. Die Ausdifferenzierung der Geräteformen, insbesondere der Trichter, lässt sich so mit dem Bedürfnis begründen, zumindest in der Frühphase des Grammophons, etwa zwischen 1900 und 1914, einen visuellen Ersatz für das fehlende Orchester und die fehlenden Sänger zu schaffen.

Aufgrund der hohen Anschaffungskosten richtete sich der Markt für Musikmedien zunächst an zahlungskräftige Käuferschichten. Die Aneignung durch das Bürgertum vollzog sich jedoch nur langsam und wurde in den Anfangsjahren von einer großen Skepsis begleitet, da das mechanische Gerät zunächst als Fremdkörper im Haushalt angesehen wurde. Musikalische Bildung war in den Augen des Bürgertums eng verbunden mit Opern- und Konzertbesuchen sowie dem Ideal der Hausmusik, während der reproduzierten Musik auf Tonträgern der Ruf eines für die breite Masse herge-

8 Vgl. Gauß 2009, 53.

9 Vgl. Ross 2008, 45.

10 Vgl. Jüttemann 2007, 109. 
stellten Konsumguts anhaftete. Werbestrategien zielten deshalb darauf ab, Musikmedien mit den bürgerlichen Idealen zu harmonisieren. Unternehmen wie Victor, Columbia oder die Deutsche Grammophon setzten dabei auf das „Starsystem“ und warben gezielt mit bekannten Künstlern wie Enrico Caruso, deren Musik die Käufer mit dem Erwerb von Grammophon und Schallplatten zu Hause hören können sollten. Caruso war der erste Grammophonkünstler, der mit einer Schallplattenaufnahme (,Vesti la giubba“ aus Ruggero Leoncavallos Oper Pagliacci aus dem Jahr 1904) über eine Million Exemplare verkaufte. ${ }^{11}$ Edison versuchte zudem, den Phonographen zum Musikinstrument zu stilisieren. ${ }^{12}$ Die Modelle hießen z. B. „Opera“ oder „Concert“.

Die Grammophonindustrie reagierte auf die Reserviertheit der bürgerlichen Schichten mit neuen Formen. So wurden Grammophone in größere Gehäuse eingebaut, in die auch die Schallführung, d.h. die Trichter integriert wurden. Diese Kabinette waren unauffälliger als die Tischapparate, da sie wie Möbelstücke aussahen. Edison stellte ab 1913 überhaupt keine tragbaren Koffergeräte mehr her, sondern bot seine Phonographen ausschließlich in Form von Schatullen und voluminösen Kabinetten an, die nicht wie technische Apparate, sondern wie repräsentative Einrichtungsgegenstände aussahen. Erst durch die Camouflage in Kabinettschränken, die sich an epochentypischen Möbeln orientierten, stießen Musikmedien auf eine breitere Akzeptanz des Bürgertums, da diese den Blick auf die Musikquelle verdeckten und so schließlich eine repräsentative Funktion übernehmen konnten, die zuvor dem Klavier vorbehalten war.

Die skeptische Haltung des Bürgertums bezog sich jedoch auch auf die Funktion des Apparates, Musik zu reproduzieren. Gebildete Schichten vertraten die Meinung, dass das „von allen Spuren der Vermittlung befreite Hören [...] die höchste Form des Musikkonsums“ sei, ${ }^{13}$ und dementsprechend schwer taten sie sich mit der Aneignung der Musikapparate. Edison stellte daher vor allem die gehobene Klangqualität seiner Apparate in den Vordergrund, die Musik nicht nachahmen, sondern „natürlich“ wiedergeben können sollten. ${ }^{14} 1913$ brachte Edisons North America Phonograph Company zu diesem Zweck ein eigenes Schallplattenformat, die Diamond Disc, auf den Markt. Diese hatte eine Abspieldauer von vier Minuten und wies weniger Störgeräusche auf als Grammophonplatten und Wachswalzen. Da die Aufnahmeund Wiedergabetechnik nach wie vor auf der Tiefenschrift beruhte, konnten Diamond Discs allerdings nur auf Edison-Geräten wiedergegeben werden.

Um die Diamond Disc bekannt zu machen, veranstaltete Edison ab 1915 sog. „Tone Tests“, mit denen er potentielle Käufer davon überzeugen wollte, dass die verbesserte Klangwiedergabe seiner Apparate dem Hörerlebnis eines Konzerts gleichkam. ${ }^{15}$ Die

11 Vgl. Hiebler 2013, 64.

12 Vgl. Thompson 1995, 142.

13 Gauß 2009, 289.

14 Vgl. Gitelman 2006, 71; vgl. Barnett 2006, 307.

15 Vgl. Thompson 1995, 149, 152. 
„Tone Tests“ waren öffentliche Konzerte, deren Ablauf einem strengen Muster folgte: Nach den einleitenden Worten eines Edison-Vertreters sang zunächst eine Sängerin synchron mit ihrer Gesangsaufnahme eine Arie. Es folgten ein oder zwei Solomusiker, die zu reproduzierter Orchesterbegleitung spielten. Am Schluss sang wieder die Künstlerin, zumeist ein Volkslied. Während des Stückes wurde die Bühne verdunkelt und das Publikum gebeten, allein durch intensives Hören zu entscheiden, ob die Sängerin persönlich oder ihre Reproduktion (in den Tone Tests Re-Creation genannt) zu hören war. Nach der erneuten Erhellung des Saals war die Sängerin, die viele Anwesende zu hören geglaubt hatten, von der Bühne verschwunden, stattdessen war lediglich die Musikaufnahme zu hören. Der unbemerkte Übergang wurde möglich, weil der Phonograph von Anfang an mitlief und die Nebengeräusche daher durchgängig $\mathrm{zu}$ hören gewesen waren und die Sängerinnen außerdem dazu angehalten wurden, möglichst ähnlich wie ihre Aufnahme zu singen.

Die „Tone Tests“ sollten Musikaufnahmen zum einen vom schlechten Image der billigen Kopie befreien und zum anderen ein eigenständiges Klangerlebnis etablieren, als imaginäre Aufführung, die einem realen Konzertbesuch in nichts nachstehen sollte. Da die Diamond Discs mit Grammophonen nicht kompatibel waren und die Musikauswahl im Vergleich zu Schellackplatten recht klein war, hatte die neue Technik zwar nur wenig Chancen, sich auf dem Markt zu behaupten. Die Idee der fidelity oder „klangtreuen“ Musikwiedergabe entwickelte sich jedoch zu einer langlebigen Werbestrategie der Hersteller.

Den entscheidenden Übergang von Tischgeräten zu Grammophon-Kabinetten kurz vor Ausbruch des Ersten Weltkriegs illustriert Thomas Mann eindrücklich im letzten Teil seines Romans Der Zauberberg. Bei dem für den Klaviersalon des Sanatoriums Berghof neu angeschafften Grammophon handele es sich ausdrücklich nicht um ein ,armselige[s] Kurbelkästchen [...], das ehemals wohl, Drehscheibe und Griffel obenauf, Anhängsel eines unförmigen Trompetentrichters aus Messing [...] anspruchslose Ohren mit näselndem Gebrüll erfüllte. "16 Stattdessen sei der Apparat wohlverwahrt in einen „mattschwarzen gebeiztem Schrein“ eingelassen, der sich mittels eines Deckels öffnen lasse und zudem mit einem elektrischen Antrieb ausgestattet sei. Ähnlich wie ein Edison-Vertreter bewirbt der Stifter des Apparats, Hofrat Behrens, das Schränkchen nicht als technisches Gerät, sondern als Musikinstrument:

Das ist kein Apparat und keine Maschine [...], das ist ein Instrument, das ist eine Stradivarus, eine Guarneri [...]. Deutsches Fabrikat, wissen Sie. Wir machen das mit Abstand am besten. Das treusinnig Musikalische in neuzeitlich-mechanischer Gestalt. Die deutsche Seele up to date. ${ }^{17}$

Die Tauglichkeit des Grammophons zum bürgerlichen Zeitvertreib wird gerade nicht über die Wiedergabetechnologie definiert, sondern paradoxerweise über das

16 Mann 1924/1981, 894.

17 Mann 1924/1981, 895. 
Möbel, in dem sich das Gerät befindet. Manns Protagonist Hans Castorp ist von dem Musikmöbel dermaßen eingenommen, dass er sich zum Hüter der grammophonen „Literatur“ (sprich der Schallplatten) erklärt und sich nachts heimlich in den Salon schleicht, um „zu musizieren“ und sich - ein Audiophiler erster Stunde - der „Fülle des Wohllauts“ hinzugeben. ${ }^{18}$ Erst das als Möbelstück und Musikinstrument inszenierte Grammophon und die zur „klangtreuen“ Wiedergabe stilisierte Musikreproduktion ermöglichen es dem Bürgertum, technische Musikwiedergabe als „objektiviertes kulturelles Kapital“ anzuerkennen. ${ }^{19}$ Vor allem der Werkstoff Holz fungierte hier als eine Art materielle Vermittlungsinstanz, die den technischen Fremdkörpern die Integration in den privaten Wohnraum ermöglichte.

Tonträger veränderten die Praktiken des Musikhörens und des Musikkonsums grundlegend: Da Musikstücke beliebig oft wiederholt werden konnten, wurden die Menschen eines Stücks schneller überdrüssig und dadurch dazu angeregt, es bald durch neue Musik zu ersetzen. Schallplatten wurden für Musikrichtungen, die wie der Jazz nur bedingt auf schriftlicher Notation beruhten, buchstäblich zur musikalischen „Literatur“ und erreichten Gesellschaftsschichten, die bis dahin nur klassische Musik hörten. Gleichzeitig verlangte das Gerät aber auch die Aufmerksamkeit und Zuwendung seiner Hörer und beförderte auf diese Weise die Ausbildung phonografischer Medienpraktiken: die Platten mussten gereinigt, aufgelegt oder umgedreht, die Nadel richtig platziert und regelmäßig ausgetauscht werden. Musikmedien, die wie Phonograph und Grammophon auf Tonträgern mit einer relativ kurzen Abspieldauer basieren, erforderten aktive Hörer, die sich zum einen dem Gerät selbst, zum anderen aber auch der Organisation des zeitlichen Ablaufs der Musikwiedergabe widmen musste, damit die Pausen beim Wechseln der Platten nicht zu lang wurden. Diese Musikpraktiken änderten sich allerdings mit der Verbreitung des Radios Mitte der 1920er Jahre.

\section{Radio}

Das Medium Radio geht historisch auf die um 1900 entwickelte Technik der drahtlosen Telegrafie zurück. Während die Funktechnik zunächst im Schiffsverkehr und später auf den Schlachtfeldern des Ersten Weltkriegs zum Einsatz kam, wandelte sich das Radio durch die Einrichtung öffentlicher Sendeanstalten (in Deutschland im Herbst 1923) zum Unterhaltungs- und Informationsmedium Rundfunk mit einer rasch wachsenden Hörerschaft. Die Besonderheit des Radios bestand vor allem darin, ein buchstäblich trägerloses Medium zu sein. Akustische Signale werden nicht mit Hilfe materieller Speichermedien, sondern allein durch die Übertragung elektroma-

18 Mann 1924/1981, 895, 903.

19 Vgl. Bourdieu 2005, 53. 
gnetischer Wellen wiedergegeben - eine Medientechnik, die der Radiostimme den Anschein von Körperlosigkeit verlieh.

Bereits in seinen Anfängen sendete der Rundfunk Nachrichten, Reportagen, Konzerte und Musik direkt in die Wohnungen und veränderte dadurch das Verständnis von Öffentlichkeit. Die Teilhabe am öffentlichen Geschehen war nun nicht mehr an eine physische Anwesenheit gebunden, sondern konnte auch von zuhause aus erfolgen. Die Rhetorik des Radios zeichnet sich durch das Versprechen aus, die Hörerschaft zu akustischen Zeugen räumlich entfernter Ereignisse werden zu lassen - und zwar, anders als im Fall von Zeitungen, simultan. Dadurch suggeriert der Rundfunk den Rezipienten, wichtige Ereignisse unmittelbar miterleben zu können. Metaphern für das Radio wie das „Ohr zur Welt“20 drücken die Faszination dieser Gleichzeitigkeitserfahrungen aus, die das neuen Medium ermöglichte. Dass nun zudem viele Hörer simultan am gleichen Informationsfluss teilhaben konnten, machte das Radio zum ersten „Massenmedium“.

Wie Carsten Lenk in seiner medienkulturwissenschaftlichen Arbeit über die Entstehung des Rundfunks herausgearbeitet hat, spielte bei der ,Domestizierung“ und Aneignung des Rundfunks im Wohnraum die „Camouflage des Technischen“ eine bedeutende Rolle. ${ }^{21}$ Durch seine Programmvielfalt richtete sich das Radio an alle Familienmitglieder und wurde meist im zentralen Raum des Hauses aufgestellt, gewöhnlich der Wohnstube oder Wohnküche. Die Gestaltung des Apparats orientierte sich demgemäß an der bereits vorhandenen Wohnungseinrichtung. Alle technischen Elemente wurden in einem Holzgehäuse untergebracht und so dem Anblick entzogen. Zu Anfang wiesen Radios keine eigenständige Formgebung auf, sondern waren an vertraute Objekte, wie bspw. Kaminuhren, angelehnt. Mit der Integrierung des Lautsprechers in das Empfängerteil bildete sich die Pultform im Hochformat sowie ab 1934 die flachliegende Kastenform aus. Das Radio verfügte über eine Schauseite, die größtenteils von der Lautsprecherbespannung eingenommen wurde, und eine Rückseite, von der aus die Röhren gewechselt werden konnten. Ein weiteres optisches Merkmal auf der Gehäusefront bildete die Senderskala.

Die Vorstellung eines unmittelbaren „Dabeiseins“, die durch das simultane Hören entstand, fand auf visueller Ebene ihr Gegenstück in der Gestalt der Senderskala, auf der zumeist die Städtenamen der Sendestationen verzeichnet waren. In den 1920er Jahren mussten Radiohörer die Sender allerdings zunächst noch mühsam suchen. Einmal gefundene Sender wurden daher zumeist nicht mehr verstellt - stattdessen begnügte man sich mit dem lokalen Radioprogramm. ${ }^{22}$ Ein erster Fortschritt bei der Sendersuche zeigte sich in den skalierten Drehknöpfen und Mikroskalen, die eine visuelle Orientierung boten. Noch mehr Komfort ergab sich durch die Einfüh-

20 Vgl. Bleicher 2000.

21 Vgl. Lenk 1997, 110-114.

22 Vgl. Lenk 1997, 114. 
rung einer feststehenden Skala, die durch mechanische Übersetzungen zwischen Skalenknopf und Drehkondensatoren das Einstellen verschiedener Sender erlaubte. Im Jahr 1933 wurden erstmals Radios in Flachbauformen auf der Funkausstellung vorgestellt, die eine linear angeordnete Stationsskala aufwiesen. Mit solch einem Radio kamen Städte aus aller Welt in die Wohnung und visualisierten die damals neuartige Möglichkeit der Vernetzung ferner Orte miteinander. ${ }^{23}$ Als zentrales Element auf der Gehäusefront symbolisierte die Skala die einfachere Bedienung und regte - nicht zuletzt durch die verheißungsvollen Namen ausländischer Städte - zu einem häufigen Senderwechsel an. ${ }^{24}$

Der Aufstieg des Rundfunks zum ersten elektronischen Massenmedium geht einher mit der massenhaften Herstellung der Empfangsgeräte; eine Entwicklung, die in Deutschland vor allem mit dem sogenannten „Volksempfänger“ verbunden ist. Nach der Machtübernahme der Nationalsozialisten forderte der neue Reichsminister für Volksaufklärung, Joseph Goebbels, dass jeder Haushalt über ein Rundfunkempfangsgerät verfügen sollte, um darüber politische Einflussnahme üben zu können. Zu diesem Zweck wurde ein Wettbewerb zur Entwicklung eines Volksempfängers ausgeschrieben. Der prämierte Entwurf stammte vom Architekten Walter Maria Kersting, die elektrische Konstruktion von Otto Grießing, einem Ingenieur der Firma Seibt. Das unter der Modellbezeichnung „VE 301“ vertriebene Radio (die Bezeichnung ist eine Anspielung auf das Datum der Machtergreifung) wurde schließlich als Gemeinschaftsprodukt von 28 Radiogeräteherstellern und 50 Zulieferfirmen produziert. Das Gerät konnte mit Gleichstrom, Wechselstrom und Batterien betrieben werden und kostete anfangs 76 Reichsmark, womit es der günstigste Rundfunkempfänger auf dem Markt war. Der niedrige Preis wurde erreicht, indem man das Gerät in wenigen Produktionsschritten arbeitsteilig herstellte und alle Einzelteile standardisierte. Es war mit einem Einkreiser und drei Röhren ausgestattet, wodurch das Hören weit entfernter Auslandssender von vornherein unmöglich war, da man dazu mindestens vier Röhren benötigte. Das Gehäusematerial bestand aus dem neuen und preiswerten Pressstoff Bakelit. Anders als die Markenradios seiner Zeit hatte der VE 301 keine Senderskala, sondern lediglich drei Bedienknöpfe (An/Aus, Suchknopf und Wellenbereich). Der Volksempfänger ist aufgrund seiner massenhaften Verbreitung zum visuellen Symbol eines historischen Zeitabschnitts geworden. Die Gehäusegestaltung erfüllte nicht einfach nur den Zweck der Oberflächenverschönerung, sondern an ihr lässt sich ebenso die politische Einflussnahme des NS festmachen. Im VE 301 materialisiert und manifestiert sich der Missbrauch des Rundfunks für nationalsozialistische Propaganda und Gleichschaltung der Bevölkerung.

Spielte der Rundfunk während des Dritten Reichs eine bedeutende Rolle als Unterhaltungs- und Propagandamedium, erreichte er in den 1950er Jahren seinen

23 Vgl. Fickers 2006, 99.

24 Vgl. Friemert 1993, 84. 
Höhepunkt als Unterhaltungs- und Informationsmedium im häuslichen Umfeld, bevor ab den 1960er Jahren der Fernseher diese Funktion zu übernehmen begann. Im Rahmen internationaler Neuregelungen der Rundfunkfrequenzen wurde ab 1950 auf UKW gesendet. Die auf Frequenzmodulation (FM) basierende UKW-Technik war weniger störungsanfällig und bot eine bessere Tonqualität. Ein weiterer Fortschritt war die Transistortechnik, durch die Radiogeräte ab Mitte der 1950er Jahre wesentlich kleiner und mobiler wurden. ${ }^{25}$ Die Rhetorik des Radios wandelte sich parallel mit dessen technischen, medialen und kulturellen Entwicklungen. In der Frühphase der Radiophonie hatte die Faszination des Mediums primär darin bestanden, überhaupt akustische Signale empfangen zu können, während der Inhalt der Sendungen eine untergeordnete Rolle spielte. Mit dem Ausbau der Sendeanstalten und Programme ermöglichte das Radio (auch dank einer verbesserten Bedienbarkeit), Sender schneller zu wählen und zu wechseln. Eben diese Funktion unterdrückte der massenhaft verkaufte Volksempfänger, dessen technische Ausstattung dazu anhielt, ausschließlich deutsche Sender zu hören. Aufgrund der Konkurrenz durch das Fernsehen wandelte sich das Radio in der zweiten Hälfte des 20. Jahrhunderts zum „Nebenbeimedium“ und zur akustischen Kulisse.

\section{Langspielplatte, Single und Stereoanlage}

In den 1950er und 60er Jahren kam es zu weitreichenden Neuerungen im Bereich der Tontechnik, die sich gleichermaßen auf Musikproduktion und -rezeption auswirkten. Neben dem modernen Schallplattenspieler und der Langspielplatte, die Musikaufnahmen stereophon und mit einer erheblich verbesserten Klangqualität wiedergeben konnten, kamen das Tonband und die Audiokassette als neue Tonträger für den Privatgebrauch in den Handel. Bei den Abspielgeräten begannen modulare Stereoanlagen, die zuvor gebräuchlichen Gerätekombinationen wie z.B. Musiktruhen abzulösen. Musikstile wie Rock'n'Roll, Rhythm \& Blues und Beatmusik, die sich während dieser Zeit international verbreiteten, sind bis heute untrennbar mit diesen neuen Musikmedien verbunden.

Bereits in der Ära von Phonograph und Grammophon versuchten Gerätehersteller, die Tonqualität der analogen Schallaufzeichnung und -wiedergabe durch technische Innovationen - etwa durch die Einführung von elektrischen Schalldosen, Mikrofonen und Lautsprechern Mitte der 1920er Jahre - zu verbessern. Dennoch war die Schallwiedergabe aufgrund einer begrenzten Frequenzbandbreite von einem markanten Eigenklang und störenden Nebengeräuschen begleitet. Zudem musste die Stahlnadel des Tonabnehmers häufig gewechselt werden, und auch die äußerst emp-

25 Vgl. Weber 2008, Kap. 3. 
findlichen Schallplatten nutzten sich relativ schnell ab. Sie bestanden hauptsächlich aus dem teuren Naturmaterial Schellack, weshalb bereits 1930 ein alternativer, synthetischer Werkstoff entwickelt wurde: Schallplatten aus Polyvinylchlorid (PVC), kurz Vinyl genannt, waren robuster, erreichten eine wesentlich längere Abspieldauer und wiesen deutlich weniger Störgeräusche auf. Neue Tonträger konnten sich jedoch vor dem Zweiten Weltkrieg mangels der Verbreitung geeigneter Abspielgeräte nicht durchsetzen.

1948 stellte das Plattenlabel Columbia die Long Play record (LP) vor, eine 12 Zoll große Vinylschallplatte mit Mikrorillenaufzeichnung, die eine Aufnahmekapazität von 23 Minuten je Seite bei einer Abspielgeschwindigkeit von 33 1/3 $\mathrm{min}^{-1}$ besaß. Ein knappes Jahr später präsentierte RCA Victor das Singleformat, eine nur 7 Zoll messende Vinylschallplatte mit großem Mittelloch und einer maximalen Abspieldauer von fünf Minuten bei $45 \mathrm{~min}^{-1} .{ }^{26}$ Der als „Battle of the Speeds“ bezeichnete Formatstreit kulminierte schließlich in dem Bau von Plattenspielern, die beide Geschwindigkeiten abspielen konnten. Die Single erlangte ihre Popularität vor allem durch den Verkauf von Hits und die Verwendung in Jukeboxes, während die LP im Bereich der Popmusik die Grundlage für die sich neu entwickelnde Form des Konzeptalbums schuf, bei dem die einzelnen Musiktitel eine stärkere inhaltliche bzw. musikalische Einheit bildeten. Für die Hörer klassischer Musik bedeutete die LP, endlich ganze Sinfonien - oder zumindest längere Sätze ohne Unterbrechung durch Seitenwechsel hören zu können.

Rund zehn Jahre nach dem Übergang von Schellack zu Vinyl veränderten sich auch die technischen Verfahren der Musikaufnahme und -wiedergabe durch die Stereotechnik. Stereophonie bedeutet räumliche Klangwiedergabe und wird technisch durch eine mehrkanalige Signalübertragung auf mindestens zwei Lautsprecher erreicht, durch die ein räumlich plastisches Klangbild erzeugt wird. ${ }^{27}$ Im Zusammenhang mit der 1958 eingeführten Stereoschallplatte warben Gerätehersteller mit dem Begriff High Fidelity (HiFi) auf den Plattencovern. Die Auszeichnung der ,Klangtreue“ existierte bereits in den 1930er Jahren als Qualitätsmerkmal für elektrifizierte Phonoobjekte. ${ }^{28}$ Seit den 1950er Jahren werden mit dem Begriff „HiFi“ hochwertige Klangaufzeichnungen bezeichnet. In der sogenannten HiFi-Norm (DIN 45500) aus dem Jahr 1967 wurden schließlich tontechnische Mindestanforderungen an Audiowiedergabegeräte verbindlich festgeschrieben. Diese betreffen das Frequenzspektrum, den Dynamikumfang und die Stereowiedergabe. Alle Geräte, die sich unterhalb eines bestimmten technischen Qualitätsniveaus befanden, durften fortan nicht länger als „High Fidelity“ bezeichnet werden. ${ }^{29}$ Da einzelne Komponenten nun elektroakustisch

26 Vgl. Hiebler 2005, 220.

27 Vgl. Wicke u. a. 2007, 694.

28 Vgl. Gauß 1998, 70.

29 Vgl. Nisius 1979, 18. 
miteinander kompatibel wurden, trug die HiFi-Norm wesentlich zur Verbreitung der modular aufgebauten Stereoanlage bei. Diese ermöglichte es Konsumenten, sich ein Ensemble von Wiedergabegeräten nach ihren eigenen Vorstellungen zusammenzustellen.

Mediengestütztes Musikhören wurde seit der Verbreitung von Schallreproduktionsmedien am Hörerlebnis des Konzerts bemessen. Dies verdeutlichen nicht zuletzt zeitgenössische und oft in der Musikwissenschaft geprägte Ausdrücke wie „Musik zweiten Grades“, „Tonträger-Musik“ und „Übertragungsmusik“. ${ }^{30}$ Bis in die 1960er Jahre hinein herrschte die Meinung vor, je neutraler, d.h. je weniger der Klang des Reproduktionsmediums zu hören sei, umso näher komme die Aufzeichnung dem Schallereignis bei der Aufnahme. ${ }^{31}$ Die Hersteller von HiFi-Technik warben etwa für die Natürlichkeit und den transparenten Klang ihrer Geräte. Ihre Emanzipation vom ewigen Vergleich zwischen Original und Reproduktion verdankt die Schallplatte einem neuen Aufnahmeverfahren in der Musikproduktion - dem Magnettonband. In der Nachkriegszeit etablierten sich Tonbandmaschinen als „Brückenmedium“ für die Aufnahmen im Tonstudio. Der erste entscheidende Vorteil bestand zunächst darin, dass Tonbänder überschrieben werden und Musikaufzeichnungen dadurch direkt nach der Aufnahme überprüft und gegebenenfalls wiederholt werden konnten. Zweitens konnten Tonbandaufnahmen geschnitten werden und im Mehrspurverfahren erfolgen. Da ein Aufnahmeband aus zunächst zwei, dann vier und später bis zu 24 und mehr Spuren bestand, war es möglich einzelne Instrumente und Singstimmen separat einzuspielen und erst in einem weiteren Verarbeitungsschritt, der Tonmischung, zu einem Masterband zusammenzuführen (Mehrspursynchron-Verfahren). Die einzelnen Tonspuren ließen sich so nachbearbeiten, in ihrer Lautstärke regulieren und mit Klangeffekten wie z. B. künstlichem Nachhall belegen. Durch die neue Produktionstechnik entstanden Musikaufnahmen, die nicht länger als Reproduktion einer originären Aufführung aufgefasst wurden, sondern gesteigerte, auf die häusliche Rezeption ausgelegte Hörerlebnisse ermöglichten. Das Tonstudio entwickelte sich zunehmend von einem tontechnischen Labor der neutralen, möglichst „klangtreuen“ Schallaufzeichnung zu einer Klangwerkstatt bzw. einem Meta-Musikinstrument zur Herstellung von ästhetischen Formen, die ein Produkt der Wechselwirkung zwischen Musikaufzeichnung und kreativer Musikbearbeitung darstellten. Folglich diente seitdem nicht mehr das Konzert als Referenzsituation für die Musikaufzeichnung, sondern die Schallplattenaufzeichnung bildete umgekehrt den neuen Standard, an dem sich Live-Aufführungen messen lassen mussten - eine Entwicklung, die der Vinylschallplatte zum Kultstatus verhalf. Als Meilensteine für die Nutzung des Mehrspurverfahrens und den kreativen Umgang mit dem Tonstudio gelten das Album

30 Vgl. Klages 2002, 2.

31 Vgl. Björnberg 2009, 108. 
Sgt. Pepper's Lonely Hearts Club Band der Beatles, die Single Good Vibrations von den Beach Boys und die Aufnahmen des Pianisten Glenn Gould. ${ }^{32}$

Die technisch-klanglichen Verbesserungen in Form von HiFi-Norm, Stereophonie und Vinylschallplatte stellten erhöhte Ansprüche an die Musikgeräte, was sich auch auf die Gerätegestaltung auswirkte. Die konservativen 1950er Jahre waren geprägt von Musikschränken und -truhen, die in erster Linie Repräsentationszwecke erfüllen sollten. HiFi-Geräte hingegen benötigten zur Stereowiedergabe zwei getrennte Lautsprecher im Raum, was die Modularisierung der Kombigeräte in Einzelkomponenten beförderte. Die Entwicklung vom Musikschrank zur Bausteinanlage hat Monika Röther in ihrem Buch The Sound of Distinction eingehend untersucht. ${ }^{33}$ Darüber hinaus hat Keir Keightley dargestellt, dass die HiFi-Stereoanlage vor allem als „maskuline“ Technologie vermarktet wurde. Der modulare Aufbau der Anlagen ermöglichte es den männlichen Kunden, sich vom Mainstream der als „feminin“ gegenderten Kombigeräte abzusetzen und durch die Kennerschaft verschiedener Gerätetypen zu profilieren. Allerdings führten die Musikanlagen im Wohnzimmer angeblich nicht selten zu Diskussionen zwischen Mann und Frau, weil das Musikhören mit der HiFi-Anlage zumeist mit hohen Lautstärken verbunden war. ${ }^{34}$ Ab dem Ende der 1950er Jahre war die Stereoanlage als Konsumobjekt zudem eng mit dem Aufkommen neuer Musikstile verbunden. Zum Image von Rock- und Beatmusik passte kein behäbiges Musikmöbel. Diese mehrheitlich jüngeren Hörer setzten sich von der älteren Generation ab und inszenierten die nun als fortschrittlich geltende und wesentlich positiver besetzte Technik der Musikreproduktion bewusst als Mittel sozialer Distinktion im Wohnraum.

Als richtungsweisend für eine neue Gestaltung von Musikgeräten gilt die Firma Braun, die in Zusammenarbeit mit der Hochschule für Gestaltung Ulm (hfg ulm) eine völlig neue Optik der Geräte etablierte. Die noch heute üblichen transparenten Plexiglasabdeckungen von Plattenspielern gehen beispielsweise auf Modelle von Dieter Rams und Hans Gugelot zurück, die - ähnlich wie schon die Nickel-in-the-SlotMachines um 1900 - die freie Sicht auf den technischen Ablauf der Musikwiedergabe ermöglichten. Die neuen Braun-Geräte wurden nicht mehr hauptsächlich aus Holz und Messing, sondern aus weißem, grauem oder schwarzem Blech gefertigt. Sie zeichneten sich durch eine klare, schnörkellose Formgebung aus, die schon bald von anderen Herstellern übernommen und zum Inbegriff modernen Designs wurde. Das Angebot an HiFi-Stereoanlagen in Westdeutschland wuchs in den 1960er und 70er Jahren stetig. Zahlreiche HiFi-Magazine und Ratgeberbücher vermittelten die nötigen technischen Kenntnisse, die beim Zusammenstellen einer Anlage erforderlich waren. Auf diese Weise entstand eine auf die optimale Wiedergabe von Klang ausgerichtete HiFi-Kultur. ${ }^{35}$

32 Vgl. Katz 2004; Kramarz 2013.

33 Vgl. Röther 2012.

34 Vgl. Keightley 1996.

35 Schröter/Volmar 2016. 


\section{Musikkassette: Mobilität und Mixtape-Kultur}

Als Gegenentwurf zur stationären Stereoanlage gilt die Einführung der Compact Cassette (CC) bzw. Musikkassette (MC) und des Kassettenrekorders 1963 von Philips. Technisch betrachtet handelt es sich bei der Musikkassette um ein Magnettonband, das von einem Kunststoffgehäuse umgeben ist. Die Rhetorik der Kassette unterscheidet sich jedoch wesentlich von der des Tonbands. Anfangs bewarb Philips den Kassettenrekorder zwar noch als akustisches Notizbuch, ${ }^{36}$ also mit ähnlichen Gebrauchsweisen wie für ein Tonband, das im privaten Bereich ebenfalls hauptsächlich für Sprachaufnahmen verwendet wurde. Die Nutzungspraktiken des Kassettenrekorders wandelten sich jedoch rasch - die Audiokassette wurde primär zum privaten Kopieren von Musik genutzt. Besonders beliebt war sie unter Jugendlichen, da sowohl die Geräte als auch Tonträger (speziell die Leerkassetten) günstiger waren als etwa Schallplatten und Stereoanlage, dabei aber leichter zu bedienen als ein Tonbandgerät.

Röther hat in ihrer Arbeit über die Objektgeschichte von Phonogeräten drei wesentliche Merkmale der Kassette im Verbund mit dem Rekorder herausgearbeitet, durch die sie sich von Schallplatte und Tonband unterscheiden: Intermedialität, Mobilität und Nutzung durch Jugendliche. ${ }^{37}$ Angeschlossen an ein Radio oder einen Plattenspieler konnte mit dem Rekorder dank der Aufnahmefunktion jederzeit spontan Musik aufgenommen werden. Kassettenrekorder waren im Vergleich zum Plattenspieler wesentlich handlicher und funktionierten auch über Batteriebetrieb, so dass sie überall hin mitgenommen werden konnten. Ein weiteres Hauptanwendungsfeld für die Kassette bildete die Nutzung im Auto. ${ }^{38}$ Dadurch war die Lieblingsmusik, unabhängig vom Radioprogramm, auch unterwegs verfügbar.

Im Unterschied zum Schallplattensammler forderte die Rhetorik der Kassette ihre Nutzer implizit dazu auf, Alben zu kopieren oder einzelne Musikstücke auf einem leeren Medium zusammenzustellen und diese nach Belieben ggf. wieder zu löschen und neu aufzunehmen. Die Kultur des „Mixtapes“, die heute allerdings oft nostalgisch als moderne Form des Liebesbriefe-Schreibens verklärt wird, beschreibt die wesentliche Nutzungsweise der Kassette. ${ }^{39}$ Die Kassette stellt außerdem ein gutes Beispiel dafür dar, dass für die Durchsetzung eines neuen Musikmediums nicht unbedingt die Tonqualität entscheidend ist. Die Kassetten und die dazugehörigen Rekorder erfüllten nämlich bis in die 1970er Jahre hinein nicht den HiFi-Standard, wodurch sie lange Zeit nicht als Komponenten für eine Stereoanlage infrage kamen. Dies änderte sich zum einen durch das Rauschunterdrückungssystem der Firma Dolby und zum

36 Vgl. Weber 2008, 167.

37 Vgl. Röther 2012, 445.

38 Vgl. Weber 2008, 175.

39 Vgl. Herlyn/Overdieck 2003. 
anderen durch die Entwicklung des Chromdioxidbands, das den Frequenzbereich der Kassette erweiterte und stereofähig machte.

Der Kassettenrekorder veränderte in den 1980er Jahren wesentlich das Verhältnis zwischen Musik und urbanem Raum. Während der Plattenspieler von den Protagonisten der frühen HipHop-Kultur vom Wiedergabemedium zum Musikinstrument umfunktioniert wurde, führten leistungsstarke tragbare Kassettenrekorder, die nicht ohne Grund als „Boombox“ oder „Ghetto Blaster“ bezeichnet wurden, zu einer neuen akustischen Aneignung des städtischen Raums. Eine buchstäblich neue Erfahrung des städtischen Raums stellt auch das private Musikhören während der eigenen Fortbewegung dar. In dieser Hinsicht steht der am Körper tragbare Mini-Kassettenrekorder Walkman von Sony seit 1979 für ein neues Lebensgefühl. Die Geschichte portabler Musikmedien hat Heike Weber in ihrer Arbeit über mobile Mediennutzung aufgearbeitet. In dieser wird neben Radio und Handy auch der Walkman behandelt. Mit dem Walkman, so Weber, werde das Hören über Kopfhörer, das bis dato ausschließlich $\mathrm{zu}$ Hause praktiziert wurde, auch unterwegs und für nahezu alle Altersgruppen zur kulturellen Praxis. ${ }^{40}$

\section{Von der Compact Disc zu Mp3: Die Digitalisierung der Musikkultur}

Eine wichtige Entwicklung in der Kultur der häuslichen Musikrezeption stellt der Wandel von der analogen zur digitalen Musikwiedergabe in den 1980er Jahren dar. Das digitale Compact-Disc-System, das die Elektronik-Konzerne Philips und Sony nach einer gemeinsamen, rund zehnjährigen Entwicklungsarbeit im Jahr 1983 vorstellten, bildete nicht lediglich eine weitere Alternativtechnologie zur Schallplatte und anderen analogen Tonträgern, sondern unterschied sich durch die Speicherung der Audiosignale im digitalen Binärcode konzeptionell radikal von den früheren Aufzeichnungsverfahren. Die Erfinder versprachen bei der Markteinführung der CD nichts weniger als die bestmögliche Klangqualität. So lautete etwa der von Philips propagierte Werbeslogan zur Vermarktung des ersten CD-Spielers „Perfect Sound Forever“. Tatsächlich verfügt der Compact-Disc-Standard über einen hohen SignalRausch-Abstand und einen großen Dynamikumfang. Durch die berührungslose optische Abtastung mittels Laser war die digitale Schallplatte zudem vor physischen Abnutzungen geschützt. Mit ihrer im Vergleich zur Langspielplatte buchstäblich kompakten Form (12 cm im Durchmesser), der glatten, silberglänzenden Oberfläche und dem hohen Kunststoffanteil passte die CD perfekt zum Neon- und Chrome-Look der

40 Vgl. Weber 2008. Siehe auch Du Gay 1997; Hosokawa 1987. 
1980er Jahre. ${ }^{41}$ Bei der CD entfiel der Seitenwechsel beim Hören von Alben, und aufgrund der Abspiellänge von bis zu 74 Minuten konnten nun auch die meisten Werke der klassischen Musik ohne Unterbrechung gehört werden. Die CD versprach zudem einen hohen Bedienkomfort. Die komplexe Funktion der optischen Abtastung wurde dabei zumeist der Sicht entzogen und im Inneren des CD-Spielers versteckt. Das Anwählen von Liedern erledigte das Laufwerk automatisch und konnte dank SkipFunktion und anderer Programm-Einstellungen (repeat song, repeat album und shuffle-Modus) per Knopfdruck gesteuert werden.

Die CD wurde speziell dafür konzipiert, die analoge Schallplatte flächendeckend abzulösen. Um die Erfolgschancen der Compact Disc zu vergrößern, vergaben Philips und Sony Lizenzen für den Bau von Abspielgeräten an andere Hersteller, so dass zum Verkaufsstart bereits zwölf CD-Player-Modelle unterschiedlicher Preisniveaus erhältlich waren. ${ }^{42}$ Die niedrigeren Herstellungs- und Versandkosten bei deutlich höheren Verkaufspreisen bescherten der Musikindustrie Traummargen. Mitte der 1990er Jahre begann die Digitalisierung allerdings ihr wahres Gesicht zu zeigen: Digitale Daten lassen sich prinzipiell verlustfrei vervielfältigen und übertragen - Vorteile, von denen etwa 15 Jahre nach der Musikindustrie zunehmend auch die Konsumenten Gebrauch zu machen begannen. Mit CD-Brennern, die als Komponenten für Personal Computer erhältlich wurden, ließ sich der Inhalt von Musik-CDs ohne Qualitätseinbußen kopieren. Ende der 1990er Jahre hatte die beschreibbare CD-R die Audiocassette praktisch als Medium der Privatkopie abgelöst. Das verlustbehaftete Audiodateiformat Mp3 erlaubte zudem eine Reduzierung des Speicherplatzbedarfes auf etwa ein Zehntel. Die Folge war - neben der Mp3-CD als Archiv- und Tauschmedium - bekanntermaßen die massenhafte Verbreitung von Musik über internetbasierte Tauschbörsen wie z.B. Napster. Die Entstehung der privaten Musikbibliothek auf Festplatte sowie die Einführung tragbarer Mp3-Player (insbesondere des Apple iPods) und Smartphones trugen maßgeblich zur Marginalisierung des physischen Tonträgers bei. ${ }^{43}$ Der als „digitales Segment“ bezeichnete Absatzmarkt für Musik über legale Download- und Streamingplattformen steigt weiter an. Dennoch machte der prozentuale Anteil von ,physischen Tonträgern' (CD, Vinyl, MC, DVD etc.) für das Jahr 2012 noch immer fast $80 \%$ des Musikverkaufs in Deutschland aus. Als paradox und gegenläufig zur Rhetorik der digitalen Musikdatei, die die allgemeine Verfügbarkeit von Musik verspricht, erscheinen auch die steigenden Verkaufszahlen von Vinylschallplatten, die 2012 den höchsten Stand seit 15 Jahren erreichten. Diese Widerständigkeit des physischen und insbesondere analogen Tonträgers hängt gleichermaßen mit einem veränderten Image der Vinylschallplatte wie mit der Tradierung bestimmter Medienpraktiken der Musikrezeption zusammen.

41 Vgl. Lingnau 1984.

42 Vgl. Lang 1996.

43 Zur Kultur- und Wissenschaftsgeschichte des MP3-Formats vgl. Sterne 2012. 


\section{Fallbeispiel: Der „kalte“ Klang des Digitalen}

Der heutige Analogkult, insbesondere in Bezug auf die Vinylschallplatte, ist teilweise auch ein Effekt der allgemeinen Digitalisierung der Musikkultur. Die Resistenz des Analogen zeigt sich bereits bei der Einführung der Compact Disc. Obwohl Philips und Sony großen Wert darauf legten, dass die CD über eine hohe Tonqualität verfügte, wurde das neue Medium jedoch gerade von HiFi-Enthusiasten äußerst skeptisch aufgenommen. In technischer Hinsicht wurden digitalen Tonträgern zwar einerseits viel Respekt entgegengebracht, aus hörästhetischer Sicht standen viele Audiophile der neuen Technologie jedoch tendenziell ablehnend gegenüber: So wurde z. B. in Fachzeitschriften wie Stereophile oder The Absolute Sound immer wieder moniert, dass der CD-Player gegenüber dem ,warmen‘ bzw. ,weichen` Sound der analogen Wiedergabegeräte einen ,kalten` bzw. ,sterilen` Klang besäße, der den Hörgenuss empfindlich störe. ${ }^{44}$ Für diese Hörer stellte die digitale Schallplatte keinen Gewinn, sondern einen Verlust an Klangqualität dar.

Die in der High-end-Kultur diskutierten Hörerfahrungen führten innerhalb kurzer Zeit zur Bildung kollektiver ästhetischer Vorstellungen über die Existenz eines spezifischen „analogen“ bzw. „digitalen“ Klangs, wobei die meisten Audiophilen der analogen Schallreproduktion klar den Vorzug gegenüber der Digitaltechnik gaben. Obwohl die hörbaren „Kinderkrankheiten“ des digitalen Mediums, die u.a. auf schlechte Digital-Analog-Wandler, technische Probleme im Prozess der Musikproduktion und eine kurzzeitige Überbetonung der Höhen in der Tonmischung relativ schnell beseitigt wurden, verbreitete und verfestigte sich unter HiFi-Enthusiasten das Vorurteil, die CD könne trotz der guten tontechnischen Eigenschaften nur eine unzureichende „natürliche“ Klangwiedergabe leisten.

Da der Markt für High-end-Audiotechnik vergleichsweise klein ist, reagierten Hersteller recht bald auf die Vorbehalte aus der audiophilen Community gegenüber der Digitaltechnik. Die Hersteller analoger Musikgeräte bestärkten die Analoganhänger in ihren Vorbehalten gegen die neue Technik und versuchten durch technische Aufrüstung von Plattenspielern und Kassettendecks den Eindruck zu vermitteln, dass man die digitale Klangwiedergabe schlicht ignorieren könne - eine Meinung, die noch heute von Verkäufern hochwertiger Schallplattenspieler vertreten wird. Auf der anderen Seite versuchten die Hersteller digitaler Wiedergabegeräte, den Bedenken der Digitalskeptiker entgegenzuwirken, und auch dies durch klangliche Verbesserungen, die oft auf technisch exzessiven Lösungen basierten. Die amerikanische Firma California Audio Labs reagierte auf den allgemeinen Vorwurf, dass CDs hart, trocken, flach, kalt usw. klängen, ebenso kreativ wie pragmatisch radikal, indem sie ihren 1986 vorgestellten CD-Spieler Tempest mit einer - aus technischer Sicht völlig unnötigen - analogen Endstufe auf Röhrenbasis auslieferte. Wie dieses Beispiel zeigt,

44 Z. B. Heister u.a. 1985; Evens 2005. 
versuchten High-end Hersteller, den Gegensatz zwischen den Hörerfahrungen und den zum Ausdruck gebrachten klangästhetischen Erwartungen der HiFi-Liebhaber mit technischen und konzeptuellen Mitteln zu harmonisieren. Tatsächlich gewannen CD-Spieler ab der zweiten Hälfte der 1980er Jahre deutlich an Akzeptanz in der audiophilen Kultur. Dennoch hält sich das Vorurteil von der Überlegenheit analoger über digitale Klangwiedergabe bis heute.

Die Rhetorik der analogen bzw. digitalen Klanglichkeit fungiert zumeist als Mittel sozialer Distinktion und Identitätskonstruktion, insbesondere zur Abgrenzung vom ,digitalen Mainstream‘, denn gegen Ende der 1980er Jahre wurden Schallplatten für die meisten Mainstream-Hörer so uninteressant, dass viele ihre vermeintlich technisch veralteten und obsolet gewordenen Schallplattensammlungen veräußerten. In den 1990er Jahren entstand so - parallel zu den sinkenden Absatzzahlen neu veröffentlichter Schallplatten - auch ein großer Markt für gebrauchte Tonträger, der musikhistorisch die gesamte Nachkriegsgeschichte umfasste. Plattenläden wurden so, mehr als je zuvor, zu Musikarchiven, die es vor allem DJs ermöglichten, sich kostengünstig mit ausgefallenem musikalischen Ausgangsmaterial für die Produktion samplebasierter Musik zu versorgen (das buchstäbliche Wühlen durch alte Plattenbestände hat sich im Ausdruck „crate digging“ niedergeschlagen). Die DJ-Kultur führte auf diese Weise zu einer doppelten Re-Auratisierung der Schallplatte in den 1990er Jahren. Erstens bildete die Schallplatte im Verbund mit Synthesizer, Sequenzer und Sampler einen zentralen Baustein samplebasierter Musikproduktion; zweitens wurde die Schallplatte durch die kreative Zweckentfremdung des Plattenspielers zum Musikinstrument der HipHop-Kultur (durch DJs wie Grand Master Flash oder Kool DJ Herc) bzw. als Medium der Rave-Kultur zum Inbegriff kreativen Musikschaffens. Die Schallplatte verdankt ihre Renaissance als Underground-Medium also gerade dem durchschlagenden Erfolg des vermeintlich ,langweiligen“ Mainstream-Mediums Compact Disc und nicht ausschließlich dem Diskurs über den unterschiedlichen Klang von Technologien. Der Rhetorik von der Überlegenheit analoger Tonträger tut dies jedoch keinen Abbruch.

\section{Literatur}

Adorno, Theodor W. (1934): Die Form der Schallplatte. In: Gesammelte Schriften, Band 19. Musikalische Schriften VI. Opern- und Konzertkritiken. Buchrezensionen. Zur Praxis des Musiklebens, Frankfurt a. M. 1984, 530-534.

Barnett, Kyle S. (2006): Furniture Music: The Phonograph as Furniture, 1900-1930. In: Journal of Popular Music Studies, 3 (18), 301-324.

Björnberg, Alf (2009): Learning to Listen to Perfect Sound: Hi-fi Culture and Changes in Modes of Listening, 1950-80. In: Derek B. Scott (Hg.): The Ashgate Research Companion to Popular Musicology. Farnhem, Surrey, 105-129.

Bleicher, Joan Kristin (2000): „Das Ohr zur Welt“. Öffentlichkeitsstrategien des frühen Hörfunks. In: Werner Faulstich/Knut Hickethier (Hg.): Öffentlichkeit im Wandel. Neue Beiträge zur Begriffsklärung. Bardowick, 132-143. 
Bourdieu, Pierre (2005): Die verborgenen Mechanismen der Macht. Hamburg.

Edison, Thomas A. (1878): The Phonograph and Its Future. In: The North American Review, 262 (126), 527-536.

Evens, Aden (2005): Sound Ideas. Music, Machines, and Experience. Minneapolis.

Fickers, Andreas (2006): Sichtbar hörbar! Radioapparat und Stadt: Knoten im vernetzten Kommunikationsraum. In: Clemens Zimmermann ( $\mathrm{Hg}$.): Zentralität und Raumgefüge der Großstädte im 20. Jahrhundert. Stuttgart, 83-104.

Friemert, Chup (1993): Radiowelten. Objektgeschichte und Hörformen. In: Wolfgang Ruppert (Hg.): Chiffren des Alltags. Erkundungen zur Geschichte der industriellen Massenkultur. Marburg, 61-104.

Gauß, Stefan (1998): Das Erlebnis des Hörens. Die Stereoanlage als kulturelle Erfahrung. In: Wolfgang Ruppert (Hg.): Um 1968. Die Repräsentation der Dinge. Marburg, 65-92.

Gauß, Stefan (2009): Nadel, Rille, Trichter. Kulturgeschichte des Phonographen und des Grammophons in Deutschland (1900-1940). Köln.

Du Gay, Paul (1997): Doing Cultural Studies. The Story of the Sony Walkman. London.

Gitelman, Lisa (2006): Always Already New. Media, History, and the Data of Culture. Cambridge, Mass.

Heister, Hanns-Werner u. a. (1985): Ästhetik der Compact Disc. Kassel u. a.

Herlyn, Gerrit/Thomas Overdieck (Hg.) (2003): Kassettengeschichten. Von Menschen und ihren Mixtapes. Münster.

Hiebler, Heinz (2005): Der Sound zwischen technischen Möglichkeiten und kulturellen Ansprüchen eine Medienkulturgeschichte der Tonträger. In: Harro Segeberg/Frank Schätzlein (Hg.): Sound. Zur Technologie und Ästhetik des Akustischen in den Medien. Marburg, 206-228.

Hiebler, Heinz (2013): Caruso auf Platte. Die Geschichte der Tonspeicherung und der Tonträger auf Platte. In: Gerhard Paul/Ralph Schock (Hg.): Sound des Jahrhunderts. Geräusche, Töne, Stimmen 1889 bis heute. Bonn, 63-68.

Hosokawa, Shūhei (1987): Der Walkman-Effekt. Berlin.

Jüttemann, Herbert (2007): Phonographen und Grammophone. Dessau.

Katz, Mark (2004): Capturing Sound. How Technology Has Changed Music. Berkeley.

Keightley, Keir (1996): „Turn It down!“ She Shrieked: Gender, Domestic Space, and High Fidelity, 1948-59. In: Popular Music 2 (15), 149-177.

Klages, Thorsten (2002): Medium und Form - Musik in den (Re-)Produktionsmedien. Osnabrück.

Kramarz, Volkmar (2013): Die Entwicklung der Recording Culture am Beispiel der Beatles in den Abbey Road Studios. In: Axel Volmar/Jens Schröter (Hg.): Auditive Medienkulturen. Techniken des Hörens und Praktiken der Klanggestaltung. Bielefeld, 269-286.

Lang, Jürgen K. (1996): Das Compact-Disc-Digital-Audio-System. Ein Beispiel für die Entwicklung hochtechnologischer Konsumelektronik. Aachen.

Lenk, Carsten (1997): Die Erscheinung des Rundfunks. Einführung und Nutzung eines neuen Mediums 1923-1932. Opladen.

Lingnau, Gerold (1984): Neuer Klang mit Silberglanz. Compact Disc kontra Langspielplatte. In: Frankfurter Allgemeine Zeitung 28. 01. 13.

Mann, Thomas (1924): Der Zauberberg. Roman. Frankfurt a. M. 1981.

Nisius, Heinz Josef (1979): HiFi hören. HiFi-Qualität? - Sie hören es! HiFi-Praxis für kritische Käufer und Fachhändler. Würzburg.

Ross, Corey (2008): Media and the Making of Modern Germany. Mass Communications, Society, and Politics from the Empire to the Third Reich. Oxford u.a.

Röther, Monika (2012): The Sound of Distinction. Phonogeräte in der Bundesrepublik Deutschland (1957-1973). Eine Objektgeschichte. Marburg. 
Rzehulka, Bernhard (1986): Abbild oder produktive Distanz? Versuch über ästhetische Bedingungen der Schallplatte. In: Matthias Fischer u. a. (Hg.): Gehörgänge. Zur Ästhetik der musikalischen Aufführung und ihrer technischen Reproduktion. München, 85-114.

Sabin, Stefana (1998): Frauen am Klavier. Skizze einer Kulturgeschichte. Frankfurt a. M. u. a.

Schröter, Jens/Axel Volmar (2016): „HiFi hören. HiFi-Praxis für kritische Käufer und Fachhändler“. Eine Fallstudie zum Problem der Analyse historischer Medienpraktiken. In: Ochsner, Beate/ Robert Stock (Hg.): Mediale Praktiken des Sehens und Hörens, Bielefeld, 149-168.

Stangl, Burkhard (2000): Ethnologie im Ohr. Die Wirkungsgeschichte des Phonographen. Wien.

Sterne, Jonathan (2003): The Audible Past. Cultural Origins of Sound Reproduction. Durham, NC u. a. Sterne, Jonathan (2012): MP3: The Meaning of a Format. Durham, N. C. u. a.

Thompson, Emily (1995): Machines, Music, and the Quest for Fidelity. Marketing the Edison Phonograph in America, 1877-1925. In: The Musical Quarterly 1 (79), 131-171.

Weber, Heike (2008): Das Versprechen mobiler Freiheit. Zur Kultur- und Technikgeschichte von Kofferradio, Walkman und Handy. Bielefeld.

Wicke, Peter u. a. (Hg.) (2007): Handbuch der populären Musik. Geschichte, Stile, Praxis, Industrie. Mainz. 
Brought to you by | Universitaetsbibliothek Basel

Authenticated Download Date | 3/8/18 11:22 AM 